Pacific Journal of Mathematics

ON GROUP ALGEBRAS OF CENTRAL GROUP EXTENSIONS 


\title{
ON GROUP ALGEBRAS OF CENTRAL GROUP EXTENSIONS
}

\author{
C. M. Edwards and P. J. Stacey
}

\begin{abstract}
If $A$ and $G$ are separable locally compact topological groups with $A$ abelian, a central group extension $G^{f}$, itself a separable locally compact topological group, of $A$ by $G$ can be defined for each Borel 2-cocycle $f$ from $G$ to $A$. The structure of the group algebras of $G^{f}$ has been studied for the case of compact $A$. In this paper structure theorems for these group algebras are obtained in the general situation.
\end{abstract}

For compact $A$ it is shown in [9] that for each element $\alpha$ of the dual group $\hat{A}$ of $A$ there exists an idempotent $R_{\alpha}$ in the centralizer $\Delta\left(L_{1}\left(G^{f}\right)\right)$ of the $L_{1}$-group algebra $L_{1}\left(G^{f}\right)$ of $G^{f}$. In [8] it is shown that $R_{\alpha}$ possesses a unique extension, also denoted by $R_{\alpha}$, to an idempotent in the centralizer $\Delta\left(C^{*}\left(G^{f}\right)\right)$ of the $C^{*}$-group algebra $C^{*}\left(G^{f}\right)$ of $G^{f}$. Moreover the family $\left\{R_{\alpha}: \alpha \in \hat{A}\right\}$ satisfies the conditions $R_{\alpha} R_{\beta}=$ $\delta_{\alpha \beta} R_{\alpha} \forall \alpha, \beta \in \hat{A}$ and $\Sigma_{\alpha \in \hat{A}} R_{\alpha}=1$, the identity operator and where the sum is the strong limit of the family of finite partial sums. However, it is shown in [3] that $\Delta\left(C^{*}\left(G^{f}\right)\right)$ is a $C^{*}$-algebra ${ }^{*}$-isomorphic to the ideal centre $\}\left(C^{*}\left(G^{f}\right)\right)$ of $C^{*}\left(G^{f}\right)$ (see [6]). Since $G^{f}$, and hence $C^{*}\left(G^{f}\right)$, is separable $\}\left(C^{*}\left(G^{f}\right)\right)$ is contained in the centre $Z\left(C^{*}\left(G^{f}\right)^{\mu}\right)$ of the Baire *(or monotone $\sigma-$ ) envelope $C^{*}\left(G^{f}\right)^{\mu}$ of $C^{*}\left(G^{f}\right)$ (see [1]). Denoting the image of $R_{\alpha}$ under the isomorphism by $r_{\alpha}$, it follows that $\left\{r_{\alpha}: \alpha \in \hat{A}\right\}$ is a family of mutually orthogonal projections in $Z\left(C^{*}\left(G^{f}\right)^{\mu}\right)$ such that $\Sigma_{\alpha \in \hat{A}} r_{\alpha}=1$, the identity in $C^{*}\left(G^{f}\right)^{\mu}$ where the sum is the least upper bound of the family of finite partial sums. Moreover for each $\alpha \in \hat{A}$, $r_{\alpha} \cdot L_{1}\left(G^{f}\right)=L_{1}\left(G^{f}, \alpha\right) \subseteq L_{1}\left(G^{f}\right) \quad$ and $\quad r_{\alpha} \cdot C^{*}\left(G^{f}\right)=C^{*}\left(G^{f}, \alpha\right) \subseteq$ $C^{*}\left(G^{f}\right)$. Hence direct sum decompositions of $L_{1}\left(G^{f}\right), C^{*}\left(G^{f}\right)$, $C^{*}\left(G^{f}\right)^{\mu}$ and $W^{*}\left(G^{f}\right)$, the $W^{*}$-group algebra of $G^{f}$, are defined.

The crucial observation allowing a theory to be developed for noncompact $A$ is that in the compact case $\hat{A} \subset L_{1}(A)$. Therefore in general, instead of studying the mapping $\alpha \rightarrow r_{\alpha}$ from $\hat{A}$ to $Z\left(C^{*}\left(G^{f}\right)^{\mu}\right)$, a mapping $\phi \rightarrow r(\phi)$ from $L_{1}(A)$ into $Z\left(C^{*}\left(G^{f}\right)^{\mu}\right)$ should be constructed. Since in general $L_{1}(A)$ does not contain idempotents, it then becomes less obvious how direct sum decompositions can be defined. The main result (Theorem 3.1) shows that such a mapping $r$ exists and has a unique extension, also denoted by $r$, to a $\sigma$-normal *-isomorphism from $C^{*}(A)^{\mu}$ into $Z\left(C^{*}\left(G^{f}\right)^{\mu}\right)$. Direct sum decompositions of $C^{*}\left(G^{f}\right)^{\mu}$ and $W^{*}\left(G^{f}\right)$ result from the abundance of idempotents in $C^{*}(A)^{\mu}$. Indeed the Fourier transform leads to a $\sigma$ - 
isomorphism between the Boolean $\sigma$-algebra of idempotents in $C^{*}(A)^{\mu}$ and the $\sigma$-algebra of Borel sets in $\hat{A}$. Therefore every Borel set $E$ in $\hat{A}$ defines a central projection in $C^{*}\left(G^{f}\right)^{\mu}$ and hence direct sum decompositions of $C^{*}\left(G^{f}\right)^{\mu}$ and $W^{*}\left(G^{f}\right)$. In particular the projections $\left\{r_{\alpha}: \alpha \in\right.$ $\hat{A}\}$ constructed in the compact case are those arising from the Borel sets in $\hat{A}$ consisting of single points.

The range $r\left(C^{*}(A)^{\mu}\right)$ of $r$ is a commutative Baire *algebra. Therefore the range $\Pi\left(r\left(C^{*}(A)^{\mu}\right)\right)$ of the restriction of a $\sigma$-normal essential representation $\Pi$ of $C^{*}\left(G^{f}\right)^{\mu}$ on separable Hilbert space is a commutative $W^{*}$-algebra (see [12]). Using this fact it is shown in $\S 4$ that every such representation possesses an essentially unique direct integral decomposition over $\hat{A}$. There exists a bijection between the set of such representations $\Pi$ of $C^{*}\left(G^{f}\right)^{\mu}$ and the set of continuous unitary representations $\pi$ of $G^{f}$ on separable Hilbert spaces. The second main result (Theorem 4.3) shows that almost all the terms in the corresponding direct integral decomposition of $\pi$ are of the form $(a, g) \rightarrow \alpha(a) \pi_{\alpha}(g)$ for some $\alpha \in \hat{A}$, where $\pi_{\alpha}$ is a projective representation of $G$ with multiplier $\alpha \circ f$.

Finally in $\$ 5$ certain results associated with the compactness of $A$ are proved. In particular it is shown that $\Sigma_{\alpha \in \hat{A}} r_{\alpha}=1$ if and only if $A$ is compact.

Results related to those in this paper, but of a rather different nature have been obtained by Insel [11].

2. Preliminaries. Throughout this paper $G$ denotes a separable locally compact topological group with unit element $e$ and $m$ denotes a left invariant Haar measure on $G$. Let $M(G)$ denote the measure algebra of $G$, let $\delta_{e}$ denote its identity and let $L_{1}(G)$ denote the $L_{1}$-group algebra of $G$. For the definitions of these and related terms the reader is referred to [10]. $L_{1}(G)$ is isometrically *-isomorphic to the closed two-sided *-ideal $M_{a}(G)$ of elements of $M(G)$ absolutely continuous with respect to $m$, by means of the mapping $\eta \rightarrow m_{\eta}$ defined for $\eta \in L_{1}(G)$ by $d m_{\eta}=\eta d m$. Let $C^{*}(G)$ denote the $C^{*}$-envelope of $L_{1}(G)$, the $C^{*}$-group algebra of $G$, and let $W^{*}(G)$ denote the $W^{*}$ envelope of $C^{*}(G)$, the $W^{*}$-group algebra of $G$. For these definitions the reader is referred to $[4,5,17]$. $C^{*}(G)$ will be identified throughout with its universal representation and therefore will be regarded as a weak* dense subalgebra of $W^{*}(G)$. The measure algebra $M(G)$ will also be identified with a subalgebra of $W^{*}(G)$ [18].

Let $C^{*}(G)^{h \mu}$ be the smallest subset of $W^{*}(G)$ containing the set $C^{*}(G)^{h}$ of self-adjoint elements of $C^{*}(G)$ and which contains the least upper bounds and greatest lower bounds of its uniformly bounded monotone sequences. Then $C^{*}(G)^{h \mu}+i C^{*}(G)^{h \mu}$ is a $C^{*}$-algebra, known as the Baire* envelope of $C^{*}(G)$ and denoted by $C^{*}(G)^{\mu}$. For 
details see [14].

There exist bijections between the families of essential representations of $L_{1}(G)$, essential representations of $C^{*}(G)$, essential $\sigma$-normal representations of $C^{*}(G)^{\mu}$ and essential normal representations of $W^{*}(G)$, the bijections being defined by restricting a given essential normal representation of $W^{*}(G)$ to $L_{1}(G), C^{*}(G)$ and $C^{*}(G)^{\mu}$ respectively. Moreover there exists a bijection $\pi \rightarrow \Pi$ from the set of continuous unitary representations of $G$ onto the set of essential representations of $L_{1}(G)$ defined for $\eta \in L_{1}(G), \xi_{1}, \xi_{2} \in H_{\pi}$, the representation space of $\pi$, by

$$
\left\langle\Pi(\eta) \xi_{1}, \xi_{2}\right\rangle=\int_{G} \eta(g)\left\langle\pi(g) \xi_{1}, \xi_{2}\right\rangle d m(g) .
$$

Each of these bijections maps primary and irreducible representations into primary and irreducible representations respectively and preserves unitary equivalence.

Let $A$ be a separable locally compact abelian group with unit element 0 , let $n$ be an invariant Haar measure on $A$ and let $\hat{A}$ be the dual group of $A$. $\hat{A}$ is discrete if and only if $A$ is compact. The Fourier transform $F$ on $L_{1}(A)$ is defined for $\phi \in L_{1}(A), \alpha \in \hat{A}$ by

$$
(F \phi)(\alpha)=\int_{A} \alpha(a) \phi(a) \operatorname{dn}(a)
$$

$F$ extends to an isometric *-isomorphism from $C^{*}(A)$ onto $C_{0}(\hat{A})$, the algebra of continuous functions on $\hat{A}$ which take arbitrarily small values outside compact sets, equipped with the supremum norm [16]. $F$ also extends uniquely to a $\sigma$-normal isometric *-isomorphism from $C^{*}(A)^{\mu}$ onto $F_{\mathfrak{B}}(\hat{A})$, the algebra of bounded Borel functions on $\hat{A}$ [12]. Both these extensions will be denoted by the same symbol $F$.

A Borel function $f$ from $G \times G$ to $A$ satisfying

$$
\begin{gathered}
f(g, e)=f(e, g)=0 \quad \forall g \in G, \\
f\left(g_{1}, g_{2}\right)+f\left(g_{1} g_{2}, g_{3}\right)=f\left(g_{1}, g_{2} g_{3}\right)+f\left(g_{2}, g_{3}\right) \quad \forall g_{1}, g_{2}, g_{3} \in G
\end{gathered}
$$

is said to be a Borel 2-cocycle from $G$ to $A$. In the special case $A=T$, the multiplicative group of complex numbers of unit modulus, a Borel 2-cocycle is said to be a multiplier on $G$. For each Borel 2-cocycle $f$ from $G$ to $A$ and each $\alpha \in \hat{A}, \alpha \circ f$ is a multiplier on $G$.

To each multiplier $\omega$ on $G$ there exists a 'twisted' convolution and involution on $L_{1}(G)$ with respect to which it forms a Banach *-algebra $L_{1}(G, \omega)$ with bounded approximate identity. $C^{*}(G, \omega), C^{*}(G, \omega)^{\mu}$ 
and $W^{*}(G, \omega)$ respectively denote the $C^{*}$, Baire* and $W^{*}$-envelopes of $L_{1}(G, \omega)$. There exist bijections between the families of essential representations of $L_{1}(G, \omega)$, essential representations of $C^{*}(G, \omega)$, essential $\sigma$-normal representations of $C^{*}(G, \omega)^{\mu}$ and essential normal representations of $W^{*}(G, \omega)$. In this case (2.1) sets up a bijection between the set of essential representations of $L_{1}(G, \omega)$ acting on a separable Hilbert space and the set of projective representations of $G$ with multiplier $\omega$ acting on a separable Hilbert space. Each of the bijections maps primary and irreducible representations into primary and irreducible representations respectively and preserves unitary equivalence. See $[7,8,9]$ for details.

Let $f$ be a Borel 2-cocycle from $G$ to $A$ and for $\left(a_{1}, g_{1}\right),\left(a_{2}, g_{2}\right) \in$ $A \times G$, let

$$
\left(a_{1}, g_{1}\right)\left(a_{2}, g_{2}\right)=\left(a_{1}+a_{2}+f\left(g_{1}, g_{2}\right), g_{1} g_{2}\right) \text {. }
$$

With this multiplication $A \times G$ is a group which possesses a separable locally compact topology, the Borel structure of which coincides with the product Borel structure and with respect to which $A \times G$ is a topological group. This group is said to be the central group extension of $A$ by $G$ corresponding to $f$ and is denoted by $G^{f}$. The measure $n \times m$ is a left invariant Haar measure on $G^{f}$ [13].

If $\mathfrak{U}$ is a complex Banach algebra, the set $\Delta(\mathfrak{H})$ of bounded linear operators $W$ on $\mathfrak{A}$ satisfying

$$
W\left(\psi_{1} \psi_{2}\right)=\left(W \psi_{1}\right) \psi_{2}=\psi_{1}\left(W \psi_{2}\right) \quad \forall \psi_{1}, \psi_{2} \in \mathfrak{A}
$$

is said to be the centralizer algebra of $\mathfrak{A}$.

Let $\mathfrak{A}$ be a $C^{*}$-algebra, let $\mathfrak{U}^{\mu}$ be its Baire* envelope and let $\mathfrak{A}^{* *}$ be its $W^{*}$-envelope. With $\mathfrak{A}, \mathfrak{A}^{\mu}$ regarded as being embedded in $\mathfrak{U}^{* *}$, the idealizer $\mathfrak{M}(\mathfrak{U})$ of $\mathfrak{A}$ is the largest $C^{*}$ - subalgebra of $\mathfrak{U} * *$ in which $\mathfrak{U}$ is an ideal. Let $\mathfrak{U}^{m}$ denote the set of self-adjoint elements of $\mathfrak{U}^{* *}$ which can be reached by increasing nets from $\mathfrak{U}^{\sim}$ the $C^{*}$-subalgebra of $\mathfrak{U}^{* *}$ obtained by adjoining the identity 1 of $\mathfrak{A}^{* *}$ to $\mathfrak{A}$. If $\mathfrak{A}_{m}=-\mathfrak{A}^{m}$, then the self-adjoint part of $\mathfrak{M}(\mathfrak{R})$ equals $\mathfrak{H}^{m} \cap \mathfrak{A}_{m}$ (1). Further $\Delta(\mathfrak{H})$ is a commutative $C^{*}$-algebra with identity and the mapping $W \rightarrow W^{* *} 1$ is a *-isomorphism from $\Delta(\mathfrak{H})$ onto the centre $Z(\mathfrak{M}(\mathfrak{U}))$ of $\mathfrak{M}(\mathfrak{A})$. Moreover $Z(\mathfrak{M}(\mathfrak{A}))=\mathfrak{Z}(\mathfrak{Q})$, the ideal centre of $\mathfrak{A}[\mathbf{2}, \mathbf{3}, \mathbf{1 5}]$. If $\mathfrak{A}$ is separable, $\mathfrak{U}^{m} \subseteq \mathfrak{U}^{\mu}, 1 \in \mathfrak{A}^{\mu}$ and hence $\mathfrak{M}(\mathfrak{H}) \subseteq \mathfrak{U}^{\mu}, \mathfrak{Z}(\mathfrak{A}) \subseteq Z\left(\mathfrak{P I}^{\mu}\right)$, the centre of $\mathfrak{A}^{\mu}$.

Throughout the paper the multiplication and involution in $W^{*}\left(G^{f}\right)$ and, for $\alpha \in \hat{A}$, in $W^{*}(G, \alpha \circ f)$ are denoted by $\cdot{ }^{*}$ respectively. 
3. The structure theorem. In this section the main theorem concerning the structure of the group algebras of $G^{f}$ is proved. It is shown that $C^{*}(A)^{\mu}$ can be embedded in the centre of $C^{*}\left(G^{f}\right)^{\mu}$. Since $C^{*}(A)^{\mu}$ possesses many idempotents, this result leads to direct sum decompositions of $C^{*}\left(G^{f}\right)^{\mu}$ and $W^{*}\left(G^{f}\right)$. The conditions under which similar decompositions of $L_{1}\left(G^{f}\right)$ and $C^{*}\left(G^{f}\right)$ also exist are examined in $\$ 5$.

The section begins with a statement of the main theorem and its corollaries.

THEOREM 3.1. For $\phi \in L_{1}(A)$ define $r(\phi)=n_{\phi} \times \delta_{e}$ where $n_{\phi} \in M(A)$ is defined by $d n_{\phi}=\phi d n$ and $\delta_{e}$ is the identity in $M(G)$. If $C^{*}\left(G^{f}\right)$ and $M\left(G^{f}\right)$ are regarded as subalgebras of $W^{*}\left(G^{f}\right)$, then the mapping $r: \phi \rightarrow r(\phi)$ extends uniquely from $L_{1}(A)$ to a $\sigma$-normal *-isomorphism from $C^{*}(A)^{\mu}$ into the centre $Z\left(C^{*}\left(G^{f}\right)^{\mu}\right)$ of $C^{*}\left(G^{f}\right)^{\mu}$.

The extension of $r$ to $C^{*}(A)^{\mu}$ will also be denoted by $r$.

COROLlaRY 3.2. For $E \in \mathfrak{B}(\hat{A})$, the $\sigma$-algebra of Borel subsets of $\hat{A}$, define $\tilde{r}(E)=r\left(F^{-1} \chi_{E}\right)$ where $\chi_{E}$ is the characteristic function of $E$, $F^{-1}$ is the inverse Fourier transform and $r$ is defined above. Then $\tilde{r}: E \rightarrow \tilde{r}(E)$ is a $\sigma$-isomorphism from $\mathfrak{B}(\hat{A})$ into the Boolean $\sigma$-algebra of central projections in $C^{*}\left(G^{f}\right)^{\mu}$.

COROllary 3.3 (i) For each Borel subset E of $\hat{A}$ with complement $E^{c}$ there exist monotone sequentially closed two-sided ideals $\tilde{r}(E) \cdot C^{*}\left(G^{f}\right)^{\mu}, \quad \tilde{r}\left(E^{c}\right) \cdot C^{*}\left(G^{f}\right)^{\mu}$ in $C^{*}\left(G^{f}\right)^{\mu}$ such that $C^{*}\left(G^{f}\right)^{\mu}=$ $\left(\tilde{r}(E) \cdot C^{*}\left(G^{f}\right)^{\mu}\right) \oplus\left(\tilde{r}\left(E^{c}\right) \cdot C^{*}\left(G^{f}\right)^{\mu}\right)$.

(ii) For each Borel subset $E$ of $\hat{A}$ with complement $E^{c}$ there exist weak* closed two-sided ideals $\tilde{r}(E) \cdot W^{*}\left(G^{f}\right), \tilde{r}\left(E^{c}\right) \cdot W^{*}\left(G^{f}\right)$ in $W^{*}\left(G^{f}\right)$ such that $W^{*}\left(G^{f}\right)=\left(\tilde{r}(E) \cdot W^{*}\left(G^{f}\right)\right) \oplus\left(\tilde{r}\left(E^{c}\right) \cdot W^{*}\left(G^{f}\right)\right)$.

(iii) The algebraic direct sums

$$
\bigoplus_{\alpha \in \hat{A}}\left(\tilde{r}(\{\alpha\}) \cdot C^{*}\left(G^{f}\right)^{\mu}\right), \quad \bigoplus_{\alpha \in \hat{A}}\left(\tilde{r}(\{\alpha\}) \cdot W^{*}\left(G^{f}\right)\right)
$$

are two-sided ideals in $C^{*}\left(G^{f}\right)^{\mu}, W^{*}\left(G^{f}\right)$ respectively.

The proof of Theorem 3.1 depends upon several results, some of which are of independent interest.

Proposition 3.4. For $\mu \in M(A)$ let $R(\mu)$ be the linear operator on $L_{1}\left(G^{f}\right)$ defined by 


$$
R(\mu) \Psi=\left(\mu \times \delta_{e}\right) \cdot \Psi \quad \forall \Psi \in L_{1}\left(G^{f}\right) .
$$

Then the mapping $R: \mu \rightarrow R(\mu)$ is an isometric *-isomorphism from $M(A)$ into $\Delta\left(L_{1}\left(G^{f}\right)\right)$.

Proof. The mapping $\mu \rightarrow \mu \times \delta_{e}$ is an isometric *-isomorphism from $M(A)$ into the centre $Z\left(M\left(G^{f}\right)\right)$ of $M\left(G^{f}\right)$. But, by Theorem 6.1 of [9], there exists an isometric *-isomorphism $X \rightarrow W_{X}$ from $Z\left(M\left(G^{f}\right)\right)$ onto $\Delta\left(L_{1}\left(G^{f}\right)\right)$ defined by $W_{X} \Psi=X \cdot \Psi, \forall \Psi \in L_{1}\left(G^{f}\right)$.

COROllaRy 3.5 For $\phi \in L_{1}(A)$ let $R(\phi)$ be the linear operator on $L_{1}\left(G^{f}\right)$ defined by

$$
R(\phi) \Psi=\left(n_{\phi} \times \delta_{e}\right) \cdot \Psi \quad \forall \Psi \in L_{1}\left(G^{f}\right) .
$$

Then the mapping $R: \phi \rightarrow R(\phi)$ is an isometric *-isomorphism from $L_{1}(A)$ into $\Delta\left(L_{1}\left(G^{f}\right)\right)$.

Proof. This follows immediately from Proposition 3.4 by regarding $L_{1}(A)$ as an ideal in $M(A)$.

Lemma 3.6 For $\phi \in L_{1}(A)$ let $R(\phi) \in \Delta\left(L_{1}\left(G^{f}\right)\right)$ be defined as above. Then $R(\phi)$ extends uniquely to an element, also denoted by $R(\phi)$, of $\Delta\left(C^{*}\left(G^{f}\right)\right)$ such that, when $C^{*}\left(G^{f}\right)$ and $M\left(G^{f}\right)$ are regarded as subalgebras of $W^{*}\left(G^{f}\right)$,

$$
R(\phi)^{* *} \Psi=\left(n_{\phi} \times \delta_{e}\right) \cdot \Psi \quad \forall \Psi \in W^{*}\left(G^{f}\right) .
$$

Proof. Let $\pi$ be an irreducible representation of $G^{f}$ on the Hilbert space $H$ and let $\Pi$ be the representation of $M\left(G^{f}\right)$ defined for $X \in M\left(G^{f}\right)$ by

$$
\left\langle\Pi(X) \xi_{1}, \xi_{2}\right\rangle=\int_{G^{\prime}}\left\langle\pi(a, g) \xi_{1}, \xi_{2}\right\rangle d X(a, g) \quad \forall \xi_{1}, \xi_{2} \in H .
$$

The irreducibility of $\pi$ implies that there exists $\alpha \in \hat{A}$ such that $\pi(a, e)=\alpha(a) 1_{H} \forall a \in A$. Therefore, by (3.0)

$$
\left\langle\left(n_{\phi} \times \delta_{e}\right) \xi_{1}, \xi_{2}\right\rangle=(F \phi)(\alpha)\left\langle\xi_{1}, \xi_{2}\right\rangle \quad \forall \xi_{1}, \xi_{2} \in H
$$

from which it follows that

$$
\left\|\Pi\left(n_{\phi} \times \delta_{c}\right)\right\|=|(F \phi)(\alpha)|
$$


Therefore, for $\Psi \in L_{1}\left(G^{f}\right)$,

$$
\begin{aligned}
\|\Pi(R(\phi) \Psi)\| & \leqq\left\|\Pi\left(n_{\phi} \times \delta_{e}\right)\right\|\|\Pi(\Psi)\| \\
& =|(F \phi)(\alpha)|\|\Pi(\Psi)\| \\
& \leqq\|\phi\|_{C^{*}(A)}\|\Psi\|_{C^{*}\left(G^{\prime}\right)}
\end{aligned}
$$

since $F$ is an isometry from $C^{*}(A)$ onto $C_{0}(\hat{A})$. By taking the supremum over all irreducible representations $\Pi$ of $L_{1}\left(G^{f}\right)$, it follows that

$$
\|R(\phi) \Psi\|_{C^{*}\left(G^{f}\right)} \leqq\|\phi\|_{C^{*}(A)}\|\Psi\|_{C^{*}\left(G^{f}\right)}
$$

Therefore $R(\phi)$ extends uniquely to a bounded linear operator, denoted by the same symbol, on $C^{*}\left(G^{f}\right)$ such that $\|R(\phi)\| \leqq\|\phi\|_{C^{*}(A)}$. Simple limit arguments show that $R(\phi) \in \Delta\left(C^{*}\left(G^{f}\right)\right)$.

The double adjoint $R(\phi)^{* *}$ of $R(\phi)$ acting on $W^{*}\left(G^{f}\right)$ is the unique weak ${ }^{*}$ continuous extension of $R(\phi)$ from $L_{1}\left(G^{f}\right)$ to $W^{*}\left(G^{f}\right)$. However, by 1.7 .8 of [17], the multiplication in $W^{*}\left(G^{f}\right)$ is weak *-continuous and so the mapping $\Psi \rightarrow\left(n_{\phi} \times \delta_{e}\right) \cdot \Psi$ is also a weak *-continuous extension of $R(\phi)$ to $W^{*}\left(G^{f}\right)$. It follows that $R(\phi)^{* *} \Psi=\left(n_{\phi} \times \delta_{e}\right) \cdot \Psi, \forall \Psi \in W^{*}\left(G^{f}\right)$.

LEMMA 3.7 The mapping $R: \phi \rightarrow R(\phi)$ from $L_{1}(A)$ into $\Delta\left(C^{*}\left(G^{f}\right)\right)$ defined in Lemma 3.6 possesses a unique extension to an isometric *-isomorphism from $C^{*}(A)$ into $\Delta\left(C^{*}\left(G^{f}\right)\right)$.

Proof. (3.2) shows that $R$ possesses a unique extension to a norm nonincreasing mapping from $C^{*}(A)$ into $\Delta\left(C^{*}\left(G^{f}\right)\right)$. Simple limit arguments show that the extension, also denoted by $R$, is a *homomorphism. For $\phi \in L_{1}(A)$,

$$
\begin{aligned}
\|\phi\|_{C^{*}(A)} & =\sup \{|(F \phi)(\alpha)|: \alpha \in \hat{A}\} \\
& =\sup \left\{\left\|\Pi\left(n_{\phi} \times \delta_{e}\right)\right\|: \Pi \in \operatorname{Irr}\left(G^{f}\right)\right\}
\end{aligned}
$$

by (3.1), where $\operatorname{Irr}\left(G^{f}\right)$ denotes the set of irreducible normal representations of $W^{*}\left(G^{f}\right)$,

$$
\leqq\left\|n_{\phi} \times \delta_{e}\right\|_{W^{*}\left(G^{\prime}\right)}=\left\|R(\phi)^{* *} 1\right\|_{W^{*}\left(G^{\prime}\right)}
$$

by Lemma 3.6,

$$
\leqq\left\|R(\phi)^{* *}\right\|=\|R(\phi)\|
$$


Hence $R$ is isometric on $L_{1}(A)$.

Let $\phi^{\prime} \in C^{*}(A)$ satisfy $R\left(\phi^{\prime}\right)=0$ and let $\left\{\phi_{\lambda}\right\}$ be a net in $L_{1}(A)$ such that, relative to the $C^{*}$-norm, $\lim \phi_{\lambda}=\phi^{\prime}$. Then, from above,

$$
\left\|\phi_{\lambda}\right\|_{C^{*}(A)}=\left\|R\left(\phi_{\lambda}\right)\right\|=\left\|R\left(\phi_{\lambda}-\phi^{\prime}\right)\right\| \leqq\left\|\phi_{\lambda}-\phi^{\prime}\right\|_{C^{*}(A)} \rightarrow 0 .
$$

It follows that $\phi^{\prime}=0$ and hence that $R$ is a *-isomorphism from the $C^{*}$-algebra $C^{*}(A)$ into the $C^{*}$-algebra $\Delta\left(C^{*}\left(G^{f}\right)\right)$. Therefore, using 1.8.1 of [4], $R$ is an isometry from $C^{*}(A)$ into $\Delta\left(C^{*}\left(G^{f}\right)\right)$.

Lemma 3.8. For $\alpha \in \hat{A}, \Psi \in L_{1}\left(G^{f}\right)$, let

$$
\left(P_{\alpha} \Psi\right)(g)=\int_{A} \alpha(a) \Psi(a, g) d n(a) \quad \forall g \in G .
$$

Then $P_{\alpha}$ is a norm nonincreasing *-homomorphism from $L_{1}\left(G^{f}\right)$ onto $L_{1}(G, \alpha \circ f)$ and $P_{\alpha}$ possesses a unique extension to a ${ }^{*}$-homomorphism from $C^{*}\left(G^{f}\right)$ onto $C^{*}(G, \alpha \circ f)$.

Proof. The calculations used in [9] to show, for the case of compact $A$, that $P_{\alpha}$ is a norm nonincreasing *-homomorphism from $L_{1}\left(G^{f}\right)$ into $L_{1}(G, \alpha \circ f)$ also apply here. To show that $P_{\alpha}$ has range $L_{1}(G, \alpha \circ f)$, let $\psi \in L_{1}(G), \phi \in L_{1}(A)$ with

$$
\int_{A} \phi(a) \operatorname{dn}(a)=1
$$

The function $\Psi$ defined for $(a, g) \in G^{f}$ by

$$
\Psi(a, g)=\overline{\alpha(a)} \phi(a) \psi(g)
$$

is an element of $L_{1}\left(G^{f}\right)$ such that $P_{\alpha} \Psi=\psi$.

The calculations used in [8] to show that, for the case of compact $A$, $P_{\alpha}$ extends uniquely to a ${ }^{*}$-homomorphism, also denoted by $P_{\alpha}$, from $C^{*}\left(G^{f}\right)$ into $C^{*}(G, \alpha \circ f)$ also apply here. However, $P_{\alpha} C^{*}\left(G^{f}\right)$ is closed in $C^{*}(G, \alpha \circ f)$ (see 1.8 .3 of [4]) and contains $L_{1}(G, \alpha \circ f)$. It follows that $P_{\alpha} C^{*}\left(G^{f}\right)=C^{*}(G, \alpha \circ f)$.

Proof of Theorem 3.1. It follows from Lemma 3.7 and the remarks at the end of $\$ 2$ that the mapping $\phi \rightarrow R(\phi)^{* *} 1$ is an isometric *-isomorphism from $C^{*}(A)$ into $Z\left(C^{*}\left(G^{f}\right)^{\mu}\right)$. Further, Lemma 3.6 shows that for $\phi \in L_{1}(A)$ 


$$
R(\phi)^{* *} 1=n_{\phi} \times \delta_{e}=r(\phi) .
$$

Since $L_{1}(A)$ is dense in $C^{*}(A)$, the mapping $\phi \rightarrow R(\phi)^{* *} 1$ is the unique extension of $r$ to $C^{*}(A)$ and will be denoted by the same symbol $r$.

Since $W^{*}\left(G^{f}\right)$ can be regarded as an algebra of operators on the universal representation space of $C^{*}\left(G^{f}\right), r$ can be regarded as a faithful representation of $C^{*}(A)$ and therefore possesses a unique extension to a $\sigma$-normal representation (also denoted by $r$ ) of $C^{*}(A)^{\mu}$. It remains to show that this extension is faithful and that its range lies inside $Z\left(C^{*}\left(G^{f}\right)^{\mu}\right)$.

Recall that the Fourier transform $F$ on $L_{1}(A)$ possesses a unique extension to a $\sigma$-normal ${ }^{*}$-isomorphism (denoted by the same symbol) from $C^{*}(A)^{\mu}$ onto the algebra $F_{\sharp}(\hat{A})$ of bounded Borel functions on $\hat{A}$. For $E \in \mathfrak{B}(\hat{A})$, the $\sigma$-algebra of Borel subsets of $\hat{A}$, let

$$
\tilde{r}(E)=r\left(F^{-1} \chi_{E}\right)
$$

where $\chi_{E}$ is the characteristic function of $E$. Since both $r$ and $F^{-1}$ are $\sigma$-normal it follows that $\breve{r}$ is a $\sigma$-homomorphism into the complete Boolean algebra of central projections in $W^{*}\left(G^{f}\right)$. It will first be shown that $\tilde{r}$ is a $\sigma$-isomorphism. To this end let $E \in \mathfrak{B}(\hat{A})$ and let $\left(\phi_{\lambda}\right)$ be a net in $L_{1}(A)$ converging to $F^{-1} \chi_{E}$ in the weak* topology of $W^{*}(A)$. Then $\chi_{E}$ is the pointwise limit on $\hat{A}$ of the net $\left(F \phi_{\lambda}\right)$. For $\alpha \in \hat{A}, \Psi \in L_{1}\left(G^{f}\right), g \in G$,

$$
\left(P_{\alpha}\left(r\left(\phi_{\lambda}\right) \cdot \Psi\right)\right)(g)=\left(P_{\alpha} R\left(\phi_{\lambda}\right) \Psi\right)(g)=\left(F \phi_{\lambda}\right)(\alpha)\left(P_{\alpha} \Psi\right)(g) .
$$

Notice that $r$ possesses a unique extension to a weak* continuous *-homomorphism (denoted by the same symbol) from $W^{*}(A)$ into $Z\left(W^{*}\left(G^{f}\right)\right)$. Using this fact, the weak* continuity of $P_{\alpha}^{* *}$ and the weak* continuity of multiplication in $W^{*}\left(G^{f}\right)$, it follows from (3.4) that, for $k \in C^{*}(G, \alpha \circ f)^{*}$,

$$
\left\langle P_{\alpha}^{* *}(\tilde{r}(E) \cdot \Psi), k\right\rangle=\lim \left\langle P_{\alpha}^{* *}\left(r\left(\phi_{\lambda}\right) \cdot \Psi\right), k\right\rangle=\lim \left(F \phi_{\lambda}\right)(\alpha)\left\langle P_{\alpha} \Psi, k\right\rangle
$$

$$
=\chi_{E}(\alpha)\left\langle P_{\alpha} \Psi, k\right\rangle \text {. }
$$

Now suppose that $E_{1}, E_{2} \in \mathfrak{B}(\hat{A})$ satisfy $\tilde{r}\left(E_{1}\right)=\tilde{r}\left(E_{2}\right)$. Let $\alpha \in E_{1}$, $\alpha \notin E_{2}$. Then, from (3.5), for $\Psi \in L_{1}\left(G^{f}\right)$,

$$
P_{\alpha} \Psi=P_{\alpha}^{* *}\left(\tilde{r}\left(E_{1}\right) \cdot \Psi\right)=P_{\alpha}^{* *}\left(\tilde{r}\left(E_{2}\right) \cdot \Psi\right)=0
$$

and since, by Lemma 3.8, $P_{\alpha}$ maps $L_{1}\left(G^{f}\right)$ onto $L_{1}(G, \alpha \circ f)$ this yields a contradiction. Hence $E_{1} \subseteq E_{2}$ and similarly $E_{2} \subseteq E_{1}$. Thus $E_{1}=E_{2}$ 
and $\tilde{r}$ is a $\sigma$-isomorphism.

To show that $r$ is an isomorphism suppose that $\phi \in C^{*}(A)^{\mu}$, $0 \leqq \phi \leqq 1, r(\phi)=0$. Then $\psi=F \phi \in F_{\mathfrak{s}}(\hat{A}), 0 \leqq \psi \leqq 1$ and the sequence $\left(1-(1-\psi)^{n}\right)$ is monotone increasing with least upper bound $\chi_{E^{\prime}}$ where $E^{\prime}=\{\alpha: \alpha \in \hat{A}, \psi(\alpha)>0\}$. By the $\sigma$-normality of $r$ and $F^{-1}$ it follows that $\tilde{r}\left(E^{\prime}\right)=0$ and therefore, from above, that $E^{\prime}=\varnothing$. Hence $\psi=0$ and. since $F$ is an isomorphism, $\phi=0$. Suppose next that $\phi \in C^{*}(A)^{\mu h},\|\phi\| \leqq 1, r(\phi)=0$. Then $\psi=F \phi \in F_{w}^{r}(\hat{A})$, the algebra of bounded real-valued Borel functions on $\hat{A},\|\psi\| \leqq 1$ and

$$
|\psi|=\left(\psi^{2}\right)^{\frac{1}{2}}=\sup \left\{1-\sum_{r=1}^{n} \frac{(2 r-3)(2 r-1) \cdots 3 \cdot 1}{(2 r)(2 r-2) \cdots 4 \cdot 2}\left(1-\psi^{2}\right)^{r}\right\} .
$$

By the $\sigma$-normality of $r$ and $F^{-1}$ it follows that $r\left(F^{-1}(|\psi|)\right)=0$ and, as above, that $|\psi|=0, \psi=0, \phi=0$. If $\phi$ is an arbitrary element of $C^{*}(A)^{\mu}$ such that $r(\phi)=0$, applying the above result to its real and imaginary part proves that $\phi=0$. Therefore $r$ is an isomorphism.

It remains to show that $r\left(C^{*}(A)^{\mu}\right) \subseteq Z\left(C^{*}\left(G^{f}\right)^{\mu}\right)$. To this end let

$$
L=\left\{\phi: \phi \in C^{*}(A)^{\mu}, r(\phi) \in Z\left(C^{*}\left(G^{f}\right)^{\mu}\right)\right\} .
$$

Let $\left(\phi_{n}\right) \subset L$ be a uniformly bounded monotone increasing sequence with least upper $\phi$. Then, by the $\sigma$-normality of $r,\left(r\left(\phi_{n}\right)\right) \subset$ $Z\left(C^{*}\left(G^{f}\right)^{\mu}\right)$ is a uniformly bounded monotone increasing sequence with least upper bound $r(\phi)$. But $Z\left(C^{*}\left(G^{f}\right)^{\mu}\right)$ is monotone sequentially closed and hence $r(\phi) \in Z\left(C^{*}\left(G^{f}\right)^{\mu}\right), \quad \phi \in L$. Therefore $L$ is monotone sequentially closed and contains $C^{*}(A)$. Hence $C^{*}(A)^{\mu}=$ $L$ and the proof is complete.

Notice that Corollary 3.2 was proved in the course of the above proof. Corollary 3.3 is an immediate consequence of the fact that $\{\tilde{r}(E): E \in \mathfrak{B}(\hat{A})\}$ is a Boolean $\sigma$-algebra of projections in $Z\left(C^{*}\left(G^{f}\right)^{\mu}\right)$.

4. Representations. Let $\operatorname{Rep}\left(G^{f}\right)$ and $\operatorname{Rep}(G, \alpha \circ f), \alpha \in \hat{A}$ respectively denote the sets of essential representations of $L_{1}\left(G^{f}\right)$ and $L_{1}(G, \alpha \circ f)$ on separable Hilbert spaces; let $\operatorname{Fac}\left(G^{f}\right)$ and $\operatorname{Fac}(G, \alpha \circ f)$ respectively denote the subsets of $\operatorname{Rep}\left(G^{f}\right)$ and $\operatorname{Rep}(G, \alpha \circ f)$ consisting of primary representations; let $\operatorname{Irr}\left(G^{f}\right)$ and $\operatorname{Irr}(G, \alpha \circ f)$ respectively denote the subsets of $\operatorname{Fac}\left(G^{f}\right)$ and $\operatorname{Fac}(G, \alpha \circ f)$ consisting of irreducible representations.

If $\Pi_{\alpha} \in \operatorname{Rep}(G, \alpha \circ f)$, then the mapping $\Psi \rightarrow \Pi_{\alpha}\left(P_{\alpha} \Psi\right)$, where $P_{\alpha}$ is defined in Lemma 3.8, on $L_{1}\left(G^{f}\right)$ is an element of $\operatorname{Rep}\left(G^{f}\right)$. The corresponding continuous unitary representation of $G^{f}$ is $(a, g) \rightarrow \alpha(a) \pi_{\alpha}(g)$, where $\pi_{\alpha}$ is the projective representation of $G$ 
corresponding to $\Pi_{\alpha}$ under (2.1). In the sequel the essential representation $\Psi \rightarrow \Pi_{\alpha}\left(P_{\alpha} \Psi\right)$ of $L_{1}\left(G^{f}\right)$ is denoted by $\alpha \otimes \Pi_{\alpha}$ and the corresponding continuous unitary representation of $G^{f}$ by $\alpha \otimes \pi_{\alpha}$. Let $\operatorname{Rep}\left(G^{f}, \alpha\right), \operatorname{Fac}\left(G^{f}, \alpha\right)$ and $\operatorname{Irr}\left(G^{f}, \alpha\right)$ respectively denote the images of $\operatorname{Rep}(G, \alpha \circ f), \quad \operatorname{Fac}(G, \alpha \circ f)$ and $\operatorname{Irr}(G, \alpha \circ f)$ under the bijection $\Pi_{\alpha} \rightarrow \alpha \otimes \Pi_{\alpha}$.

In [7] it is shown how, for compact $A$, every element of $\operatorname{Rep}\left(G^{f}\right)$ can be written as a direct sum of elements of the family $\left\{\operatorname{Rep}\left(G^{f}, \alpha\right): \alpha \in \hat{A}\right\}$. The generalization relies on the theory of direct integrals, for details of which the reader is referred to $[4,5]$. Throughout this section the commutative Baire* algebra $r\left(C^{*}(A)^{\mu}\right)$ will be denoted by $Z$.

Lemma 4.1. Let $\Pi \in \operatorname{Rep}\left(C^{f}\right)$, let $\pi$ be the corresponding continuous unitary representation of $G^{f}$ and let $\pi_{e}$ be the continuous unitary representation $a \rightarrow \pi(a, e)$ of $A$. Then $\Pi(Z)=\pi_{e}(A)^{\prime \prime}$, the Von Neumann algebra generated by $\pi_{e}(A)$.

Proof. Let $\Pi_{e}$ be the element of $\operatorname{Rep}(A)$ associated with $\pi_{e}$ and recall that $\Pi_{e}\left(C^{*}(A)^{\mu}\right)=\Pi_{e}(A)^{\prime \prime}$ (see [4], 13.3.5, [12], p. 322). A simple calculation shows that for $\phi \in L_{1}(A), \Pi_{e}(\phi)=\Pi(r(\phi))$ and hence $\Pi_{e}=\Pi \circ r$. This completes the proof of the lemma.

The first preliminary result concerning the structure of $\operatorname{Rep}\left(G^{f}\right)$ is the following.

Proposition 4.2. (i) For $\Pi \in \operatorname{Rep}\left(G^{f}\right), \Pi \in \operatorname{Rep}\left(G^{f}, \alpha\right)$ for some $\alpha \in \hat{A}$ if and only if $\Pi(Z)=\mathbf{C} 1_{H}$ where $1_{H}$ is the identity operator on the representation space $H$ of $\Pi$.

(ii) If $\alpha \neq \beta$ then $\operatorname{Rep}\left(G^{f}, \alpha\right) \cap \operatorname{Rep}\left(G^{f}, \beta\right)=\varnothing$.

(iii) $\operatorname{Fac}\left(G^{f}\right)=\cup_{\alpha \in \in_{A}} \operatorname{Fac}\left(G^{f}, \alpha\right)$.

(iv) $\operatorname{Irr}\left(G^{f}\right)=\cup_{\alpha \in \hat{A}} \operatorname{Irr}\left(G^{f}, \alpha\right)$.

Proof. (i) Lemma 4.1 shows that $\Pi(Z)$ is trivial if and only if for all $a \in A, \pi_{e}(a)=\alpha(a) 1_{H}$ for some $\alpha \in \hat{A}$. It follows that $\Pi(Z)$ is trivial if and only if $\pi=\alpha \otimes \pi_{\alpha}$ for some projective representation $\pi_{\alpha}$ of $G$ with multiplier $\alpha \circ f$ or equivalently if and only if $\Pi \in \operatorname{Rep}\left(G^{f}, \alpha\right)$ for some $\alpha \in \hat{A}$.

(ii) If $\Pi \in \operatorname{Rep}\left(G^{f}, \alpha\right) \cap \operatorname{Rep}\left(G^{f}, \beta\right)$ and if $\pi$ is the corresponding continuous unitary representation of $G^{f}$ then, for $a \in A, \alpha(a) 1_{H}=$ $\pi(a, e)=\beta(a) 1_{H}$ and so $\alpha=\beta$.

(iii) If $\Pi \in \operatorname{Fac}\left(G^{f}\right)$ then $\Pi(Z) \subseteq \Pi\left(Z\left(W^{*}\left(G^{f}\right)\right)\right)=\mathbf{C} 1_{H}$ and hence, by (i), $\Pi \in \operatorname{Rep}\left(G^{f}, \alpha\right)$ for some $\alpha \in \hat{A}$. Therefore $\Pi=\alpha \otimes \Pi_{\alpha}$ for 
some $\Pi_{\alpha} \in \operatorname{Rep}(G, \alpha \circ f)$ and, since $\Pi$ is primary, it follows that $\Pi_{\alpha}$ is also primary. It follows that $\operatorname{Fac}\left(G^{f}\right) \subseteq \cup_{\alpha \in \hat{A}} \operatorname{Fac}\left(G^{f}, \alpha\right)$ and the reverse inclusion is trivial.

(iv) The proof is similar to that of (iii).

The main result about the structure of $\operatorname{Rep}\left(G^{f}\right)$ is the following.

THEOREM 4.3. For $\Pi \in \operatorname{Rep}\left(G^{f}\right)$ there exists a positive measure $\mu \in M(\hat{A})$, unique up to measure class, and a family $\left\{\Pi^{\alpha}: \alpha \in \hat{A}\right\}$, where $\Pi^{\alpha} \in \operatorname{Rep}\left(G^{f}, \alpha\right)$ for $\mu$-almost all $\alpha \in \hat{A}$, such that $\Pi$ is unitarily equivalent to $\int_{\hat{A}}^{\oplus} \Pi^{\alpha} d \mu(\alpha)$.

Proof. $\Pi \circ r \circ F^{-1}$ is a $\sigma$-normal representation of $F_{8}(\hat{A})$ with range $\Pi(Z)$ which is a Von Neumann algebra since the representation space is separable. Moreover it is the unique $\sigma$-normal extension of its restriction to $C_{0}(\hat{A})$. By standard representation theory for $C_{0}(\hat{A})$ there exists a positive measure $\mu \in M(\hat{A})$, unique up to measure class, such that $\Pi(Z)$ is *-isomorphic to $L_{\infty}(\hat{A}, \mu)$. Using [4], 8.2.2, 8.3.2, [5] App. IV, there exists a family $\left\{\Pi^{\alpha}: \alpha \in \hat{A}, \Pi^{\alpha} \in \operatorname{Rep}\left(G^{f}\right)\right\}$ such that $\Pi$ is unitarily equivalent to $\int_{\hat{A}}^{\oplus} \Pi^{\alpha} d \mu(\alpha)$ and $\Pi(Z)$ is isomorphic to the algebra of diagonalizable operators. It remains to prove that $\Pi^{\alpha} \in$ $\operatorname{Rep}\left(G^{f}, \alpha\right)$ for $\mu$-almost all $\alpha \in \hat{A}$. It follows from Lemma 4.1 and Proposition 4.2 that this is achieved once it has been proved that, if $\pi^{\alpha}$ is the continuous unitary representation of $G^{f}$ corresponding to $\Pi^{\alpha}$, then the continuous unitary representation $\left(\pi^{\alpha}\right)_{e}$ of $A$ is primary for $\mu$-almost all $\alpha \in \hat{A}$. If $\pi^{\prime}=\int_{\hat{A}}^{\oplus} \pi^{\alpha} d \mu(\alpha)$, then by 18.7.4 of [4], $\pi^{\prime}$ is unitarily equivalent to the representation $\pi$ of $G^{f}$ associated with $\Pi$. But, by Lemma $4.1, \Pi(Z)=\pi_{e}(A)^{\prime \prime}$ and therefore the decomposition $\pi_{e}=\int_{\hat{A}}^{\oplus}\left(\pi^{\alpha}\right)_{e} d \mu(\alpha)$ is the central decomposition of $\pi_{e}$. Using 8.4.1 of [4], it follows that $\left(\pi^{\alpha}\right)_{e}$ is primary for $\mu$-almost all $\alpha \in \hat{A}$.

Remark. Let $K=\left\{k: k \in C^{*}\left(G^{f}\right)^{*}, k \geqq 0,\|k\| \leqq 1\right\}$, let $k \in K$ and let $\Pi_{k}$ be the cyclic representation of $C^{*}\left(G^{f}\right)$ on $H_{k}$ associated with $k$ (see [4], 2.4.4.). Then, according to [17], §3.1, a decomposition of $\Pi_{k}$ over $K$ corresponding to $\Pi_{k}(Z)$ can be obtained by means of a unique positive Radon measure $\nu_{k}$. Theorem 4.3 also defines a decomposition of $\Pi_{k}$ corresponding to $\Pi_{k}(Z)$, given by the measure $\mu_{k}$ on $\hat{A}$. An application of the uniqueness theorem (see [4], 8.2.4) then establishes the existence of a Borel isomorphism from $\hat{A} \backslash E$, for some Borel set $E$ satisfying $\mu_{k}(E)=0$, into $K$ which transforms $\mu_{k}$ into $\nu_{k}$. From 
Theorem 4.3, the images under this isomorphism of $\mu_{k}$-almost all of the points of $\hat{A} \backslash E$ lie in the set $\partial_{p r}^{Z}(K)=\left\{k: k \in K, \Pi_{k}(Z)=\mathbf{C} 1_{H_{k}}\right\}$, the set of $Z$-primary points of $K$. A corollary of Theorem 4.3 is therefore that the measure $\nu_{k}$ on $K$ is pseudo-concentrated on $\partial_{p r}^{Z}(K)$. Further discussion of this and related topics is not within the scope of this paper (cf. [17], §3.1).

5. The compact case. In this section the following two criteria which exhibit the compactness of $A$ are proved.

THEOREM 5.1. If the family $\{\bar{r}(\{\alpha\}): \alpha \in \hat{A}\}$ of mutually orthogonal central projections in $C^{*}\left(G^{f}\right)^{\mu}$ is defined by (3.3), then $\Sigma_{\alpha \in \hat{A}} \tilde{r}(\{\alpha\})=1$ if and only if $A$ is compact.

THEOREM 5.2. If the family $\{\tilde{r}(\{\alpha\}): \alpha \in \hat{A}\}$ of mutually orthogonal central projections in $C^{*}\left(G^{f}\right)^{\mu}$ is defined by (3.3), then

(i) $\tilde{r}(\{\alpha\}) \cdot L_{1}\left(G^{f}\right) \subseteq L_{1}\left(G^{f}\right)$ for some $\alpha \in \hat{A}$ if and only if $A$ is compact

and

(ii) $\tilde{r}(\{\alpha\}) \cdot C^{*}\left(G^{f}\right) \subseteq C^{*}\left(G^{f}\right)$ for some $\alpha \in \hat{A}$ if and only if $A$ is compact.

If $A$ is compact, the mapping $Q_{\alpha}$ defined for $\alpha \in \hat{A}, \eta \in L_{1}(G, \alpha \circ f)$ by

$$
\left(Q_{\alpha} \eta\right)(a, g)=\overline{\alpha(a)} \eta(g) \quad \forall(a, g) \in G^{f}
$$

is an isometric *-isomorphism onto a norm closed two-sided *-ideal $L_{1}\left(G^{f}, \alpha\right)$ in $L_{1}\left(G^{f}\right)$ [9]. Further, $P_{\alpha} Q_{\alpha}=1$, the identity operator on $L_{1}(G, \alpha \circ f)$ and, if $R_{\alpha}=Q_{\alpha} P_{\alpha}$, the family $\left\{R_{\alpha}: \alpha \in \hat{A}\right\}$ of projections in $\Delta\left(L_{1}\left(G^{f}\right)\right)$ satisfies $R_{\alpha} R_{\beta}=\delta_{\alpha \beta} R_{\alpha}$. A simple calculation shows that, since $\hat{A} \subset L_{1}(A)$, for $\Psi \in L_{1}\left(G^{f}\right)$

$$
R_{\alpha} \Psi=R(\bar{\alpha}) \Psi=\tilde{r}(\{\alpha\}) \cdot \Psi
$$

using the notation of $\S 3$.

The map $Q_{\alpha}$ defined by (5.1) extends uniquely to a *. homomorphism $Q_{\alpha}$ from $C^{*}(G, \alpha \circ f)$ onto a norm closed two-sided *-ideal $C^{*}\left(G^{f}, \alpha\right)$ in $C^{*}\left(G^{f}\right)$. Further, if $P_{\alpha}$ is extended, as in Lemma 3.8 , to a ${ }^{*}$-homomorphism $P_{\alpha}$ from $C^{*}\left(G^{f}\right)$ onto $C^{*}(G, \alpha \circ f)$, then $P_{\alpha} Q_{\alpha}=1$ the identity operator on $C^{*}(G, \alpha \circ f)$ and $R_{\alpha}=Q_{\alpha} P_{\alpha}$ is a projection onto $C^{*}\left(G^{f}, \alpha\right)$ [8]. By means of simple limit arguments it can be deduced from (5.2) that, if the extension of $R(\bar{\alpha})$ to an element of 
$\Delta\left(C^{*}\left(G^{f}\right)\right)$ is denoted by the same symbol, then, for $\alpha \in \hat{A}, \Psi \in$ $C^{*}\left(G^{f}\right)$

$$
R_{\alpha} \Psi=R(\bar{\alpha}) \Psi=\tilde{r}(\{\alpha\}) \cdot \Psi
$$

Lemma 5.3. If $A$ is compact then $\bigoplus_{\alpha \in \hat{A}} \tilde{r}(\{\alpha\}) \cdot W^{*}\left(G^{f}\right)$ is weak $*$ dense in $W^{*}\left(G^{f}\right)$.

Proof. It is shown in Theorem 5.5 of [9] that $\bigoplus_{\alpha \in A} R_{\alpha} L_{1}\left(G^{f}\right)$ is norm dense in $L_{1}\left(G^{f}\right)$ and hence weak dense in $W^{*}\left(G^{f}\right)$. However, by (5.2), $\bigoplus_{\alpha \in \hat{A}} R_{\alpha} L_{1}\left(G^{f}\right)=L_{1}\left(G^{f}\right) \cap\left(\bigoplus_{\alpha \in \hat{A}} \bar{r}(\{\alpha\}) \cdot W^{*}\left(G^{f}\right)\right)$, from which the result follows.

Proof of Theorem 5.1. Let $\Sigma_{\alpha \in \hat{A}} \tilde{r}(\{\alpha\})$, defined to be the least upper bound in $W^{*}\left(G^{f}\right)$ of the family $\left\{\Sigma_{\alpha \in \Lambda} \tilde{r}(\{\alpha\}): \Lambda \subseteq \hat{A}, \Lambda\right.$ finite $\}$ be denoted by $u$. If $A$ is compact then, by Lemma 5.3, there exists a net $\left(\Psi_{\lambda}\right)$ of elements of $\bigoplus_{\alpha \in \hat{A}} \bar{r}(\{\alpha\}) \cdot W^{*}\left(G^{f}\right)$ with weak* limit 1 . The weak* continuity of multiplication in $W^{*}\left(G^{f}\right)$ then implies that $(1-u)$ - $\Psi_{\lambda} \rightarrow 1-u$. However, $(1-u) \cdot \Psi_{\lambda}=0 \forall \lambda$ and thus $u=1$.

Conversely, assume that $u=1$ and let $\mu$ be a positive normalised regular Borel measure on $\hat{A}$. Let $H=L_{2}\left(\hat{A}, L_{2}(G), \mu\right)$ and for $(a, g) \in G^{f}, \xi \in H, h \in G, \alpha \in \hat{A}$, let

$$
(\pi(a, g) \xi)_{\alpha}(h)=\alpha(a)(\alpha \circ f)\left(g, g^{-1} h\right) \xi_{\alpha}\left(g^{-1} h\right) .
$$

Then $\pi$ is easily seen to be a continuous unitary representation of $G^{f}$. If $\Pi$ is the corresponding element of $\operatorname{Rep}\left(G^{f}\right)$ a simple calculation shows that for $\Psi \in L_{1}\left(G^{f}\right), \xi \in H, \alpha \in \hat{A}$,

$$
(\Pi(\Psi) \xi)_{\alpha}=L_{\alpha}\left(P_{\alpha} \Psi\right) \xi_{\alpha}
$$

where $L_{\alpha}$ is the left regular representation of $L_{1}(G, \alpha \circ f)$ defined for $\eta \in L_{1}(G, \alpha \circ f), \eta^{\prime} \in L_{2}(G)$ by

$$
L_{\alpha}(\eta) \eta^{\prime}=\eta \cdot \eta^{\prime}
$$

Since $\Pi$ possesses a unique normal extension to $W^{*}\left(G^{f}\right)$ and since, for each $\alpha \in \hat{A}, L_{\alpha}$ possesses a unique normal extension to $W^{*}(G, \alpha \circ f)$ it follows that for $\Psi \in W^{*}\left(G^{f}\right), \xi \in H, \alpha \in \hat{A}$,

$$
(\Pi(\Psi) \xi)_{\alpha}=L_{\alpha}\left(P_{\alpha}^{* *} \Psi\right) \xi_{\alpha} .
$$


Using (5.4) and Lemma 4.1 it is clear that $\Pi(Z)$ is *-isomorphic to $L_{\infty}(\hat{A}, \mu)$ and therefore $\mu$ is the measure on $\hat{A}$ corresponding to $\Pi$ through Theorem 4.3. For $\alpha \in \hat{A}$ define $\Pi^{\alpha} \in \operatorname{Rep}\left(G^{f}\right)$ on $\Pi(\tilde{r}(\{\alpha\})) H=H_{\alpha}$ for $\Psi \in W^{*}\left(G^{f}\right)$ by $\Pi^{\alpha}(\Psi)=\Pi(\tilde{r}(\{\alpha\}) \cdot \Psi)$ and notice that the hypothesis $u=1$ leads to

$$
\Pi=\bigoplus_{\alpha \in \in_{A}} \Pi^{\alpha}
$$

But, for $\alpha \in \hat{A}$,

$$
\begin{aligned}
\Pi^{\alpha}(Z) & =\Pi^{\alpha}\left(r\left(F^{-1} \chi_{\{\alpha\}}\right) \cdot Z\right)=\Pi^{\alpha}\left(r F^{-1}\left(\chi_{\{\alpha\}} \cdot F_{3 g}(\hat{A})\right)\right) \\
& =\left\{\lambda \Pi^{\alpha}(\tilde{r}(\{\alpha\})): \lambda \in \mathbf{C}\right\}=\mathbf{C 1}_{H_{\alpha \alpha} .}
\end{aligned}
$$

It follows from Proposition 4.2 and (3.5) that for each $\alpha \in \hat{A}, \Pi^{\alpha} \in$ $\operatorname{Rep}\left(G^{f}, \alpha\right)$. Therefore (5.5) describes a decomposition of $\Pi$ into a direct sum over $\hat{A}$ of elements of $\operatorname{Rep}\left(G^{f}, \alpha\right)$. Theorem 4.3 shows that $\mu$ is discrete. Hence $\hat{A}$ is discrete and $A$ is compact.

Proof of Theorem 5.2. (i) If $A$ is compact it follows immediately from (5.2) that

$$
\tilde{r}(\{\alpha\}) \cdot L_{1}\left(G^{f}\right)=R_{\alpha} L_{1}\left(G^{f}\right) \subseteq L_{1}\left(G^{f}\right) \quad \forall \alpha \in \hat{A} .
$$

Conversely, assume that $A$ is noncompact and thus that $\hat{A}$ is nondiscrete. It will be shown that

$$
L_{1}\left(G^{f}\right) \cap\left(\tilde{r}(\{\alpha\}) \cdot L_{1}\left(G^{f}\right)\right)=\{0\} \quad \forall \alpha \in \hat{A}
$$

which, because of (3.5), is a stronger result than that to be proved. For some $\alpha \in \hat{A}$, let $\left.\Psi \in L_{1}\left(G^{f}\right)\right)$ and define the mapping $d_{\Psi}$ on $\hat{A}$ by $d_{\Psi}(\beta)=P_{\beta} \Psi \forall \beta \in \hat{A}$. It follows from (3.5) that either $P_{\beta} \Psi=0$ $\forall \beta \in \hat{A}$ or $d_{\psi}^{-1}(0)=\hat{A} \backslash\{\alpha\}$. However, by Proposition 2.4 of [11], $d_{\Psi}$ is continuous and thus, if $d_{\psi}^{-1}(0)=\hat{A} \backslash\{\alpha\},\{\alpha\}$ is open. By 15.8 and 15.17 (b) of [10] this implies that $\hat{A}$ is discrete, contradicting the assumption that $A$ is noncompact. Hence $P_{\beta} \Psi=0 \forall \beta \in \hat{A}$ and, by the injective property of the Fourier transform, $\Psi=0$.

(ii) If $A$ is compact it follows immediately from (5.3) that 


$$
\tilde{r}(\{\alpha\}) \cdot C^{*}\left(G^{f}\right)=R_{\alpha} C^{*}\left(G^{f}\right) \subseteq C^{*}\left(G^{f}\right) \quad \forall \alpha \in \hat{A}
$$

Conversely, assume that $\tilde{r}(\{\alpha\}) \cdot C^{*}\left(G^{f}\right) \subseteq C^{*}\left(G^{f}\right)$ for some $\alpha \in \hat{A}$ and choose $\Psi \in C^{*}\left(G^{f}\right)$ such that $P_{\alpha} \Psi \neq 0$. It follows from (3.5) that for $\beta \in \hat{A}, P_{\beta}(\tilde{r}(\{\alpha\}) \cdot \Psi)=\delta_{\alpha \beta} P_{\alpha} \Psi$ and so, as in the proof of (i) above, it suffices to show that the mapping $\beta \rightarrow P_{\beta}(\tilde{r}(\{\alpha\}) \cdot \Psi)$ is continuous. However, given $\epsilon>0$ there exists $\Psi^{\prime} \in L_{1}\left(G^{f}\right)$ such that $\left\|\tilde{r}(\{\alpha\}) \cdot \Psi-\Psi^{\prime}\right\|<\epsilon / 4$. Then, for $\beta, \gamma \in \hat{A}$,

$$
\begin{aligned}
&\left\|P_{\beta}(\bar{r}(\{\alpha\}) \cdot \Psi)-P_{\gamma}(\bar{r}(\{\alpha\}) \cdot \Psi)\right\|_{C^{*}\left(G^{\prime}\right)} \leqq 2\left\|\bar{r}(\{\alpha\}) \cdot \Psi-\Psi^{\prime}\right\|_{C^{*}\left(G^{\prime}\right)} \\
&+\left\|P_{\beta} \Psi^{\prime}-P_{\gamma} \Psi\right\|_{C^{*}\left(G^{\prime}\right)} \\
&<\epsilon / 2+\left\|P_{\beta} \Psi^{\prime}-P_{\gamma} \Psi^{\prime}\right\|_{1}
\end{aligned}
$$

The result thus follows from the continuity of the mapping $\beta \rightarrow P_{\beta} \Psi^{\prime}$.

\section{REFERENCES}

1. C. A. Akemann, G. K. Pedersen and J. Tomiyama, Multipliers of $C^{*}$-algebras, J. Funct. Anal., 13 (1973), 277-301.

2. C. A. Akemann and G. K. Pedersen, Complications of semicontinuity in $C^{*}$-algebra theory, Duke Math. J., 40 (1973), 785-797.

3. T. B. Andersen, On multipliers and order bounded operators in $C^{*}$-algebras, Proc. Amer. Math. Soc., 25 (1970), 869-899

4. J. Dixmier, Les $C^{*}$-algèbres et leurs representations, Gauthier-Villars, Paris, 1964.

5. L Les Algèbres d'operateurs dans l'espace hilbertien, Gauthier-Villars, Paris, 1969.

6. - Ideal center of a $C^{*}$-algebra, Duke Math. J., 35 (1968), 375-389.

7. C. M. Edwards and J. T. Lewis, Twisted group algebras I, Commun. Math. Phys., 13 (1969), 119-130.

8. C. M. Edwards, $C^{*}$-algebras of central group extensions I, Ann. Inst. Henri Poincaré, 10 (1969), 229-246.

9. , The measure algebra of a central group extension, Quart. J. Math. Oxford (2), 22 (1971), $197-220$.

10. E. Hewitt and K. A. Ross, Abstract Harmonic Analysis, Springer, Berlin, 1963.

11. A. J. Insel, Maximal ideals in the group algebra of an extension, Trans. Amer. Math. Soc., 172 (1972), 195-206.

12. R. V. Kadison, Unitary invariants for representations of operator algebras, Ann. of Math., 66 (1957), 304-379.

13. G. W. Mackey, Les ensembles boreliens et les extensions des groupes, J. Math. pure et appl., 36 (1957), 171-178.

14. G. K. Pedersen, On weak and monotone $\sigma$-closures of $C^{*}$-algebras, Commun. Math. Phys., 11 (1969), 221-226.

15. , Applications of weak* semi-continuity in $C^{*}$-algebra theory, Duke Math. J., 39 (1972), $431-450$. 
16. W. Rudin, Fourier Analysis on Groups, Interscience, New York. 1962.

17. S. Sakai, $C^{*}$-algebras and $W^{*}$-algebras, Springer, Berlin, 1971.

18. M. E. Walter, $W^{*}$-algebras and non-abelian harmonic analysis, J. Funct. Anal., 11 (1972), 17-38.

Received November 20, 1973.

The QueEn's College, Oxford, England

AND

La Trobe University, Victoria, Australia. 




\section{Pacific Journal of Mathematics}

\section{Vol. 56, No. $1 \quad$ November, 1975}

Shimshon A. Amitsur, Central embeddings in semi-simple rings .......... 1

David Marion Arnold and Charles Estep Murley, Abelian groups, A, such

that $\operatorname{Hom}(A,---)$ preserves direct sums of copies of $A \ldots \ldots \ldots .$.

Martin Bartelt, An integral representation for strictly continuous linear

operators ................................... 21

Richard G. Burton, Fractional elements in multiplicative lattices......... 35

James Alan Cochran, Growth estimates for the singular values of

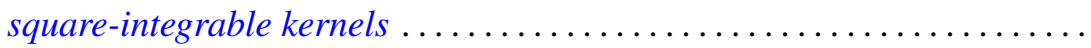

C. Martin Edwards and Peter John Stacey, On group algebras of central

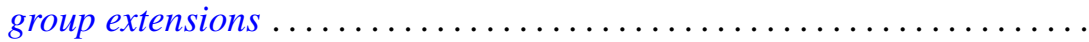

Peter Fletcher and Pei Liu, Topologies compatible with homeomorphism

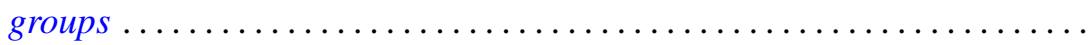

George Gasper, Jr., Products of terminating ${ }_{3} F_{2}(1)$ series ............ 87

Leon Gerber, The orthocentric simplex as an extreme simplex ............

Burrell Washington Helton, A product integral solution of a Riccati

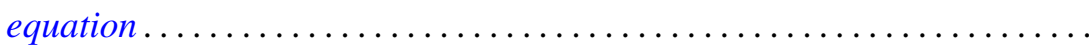

Melvyn W. Jeter, On the extremal elements of the convex cone of

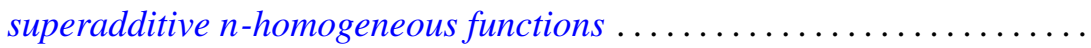

R. H. Johnson, Simple separable graphs .

Margaret Humm Kleinfeld, More on a generalization of commutative and

alternative rings. . .

A. Y. W. Lau, The boundary of a semilattice on an $n$-cell.

Robert F. Lax, The local rigidity of the moduli scheme for curves ...

Glenn Richard Luecke, A note on quasidiagonal and quasitriangular

operators .

Paul Milnes, On the extension of continuous and almost periodic functions

Hidegoro Nakano and Kazumi Nakano, Connector theory.

James Michael Osterburg, Completely outer Galois theory of perfect rings ..................................

Lavon Barry Page, Compact Hankel operators and the F. and M. Riesz

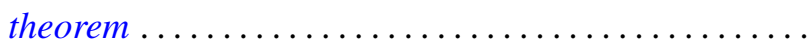

Joseph E. Quinn, Intermediate Riesz spaces..................... 225

Shlomo Vinner, Model-completeness in a first order language with a generalized quantifier.

Jorge Viola-Prioli, On absolutely torsion-free rings ..........

Philip William Walker, A note on differential equations with all solutions of integrable-square............................

Stephen Jeffrey Willson, Equivariant maps between representation 